\title{
ELECTRORESPONSIE BEHAVIOR OF POLYELECTROLYTE GELS (ACRYLIC ACID-CO-N-N' METHYLENE BISACRYLAMIDE) UNDER THE INFLUENCE OF ELECTRIC FIELD
}

\author{
Nguyen Phuong Hoai Nam* \\ Faculty of Engineering Physics and Nano-Technology, \\ College of Techology, Vietnam National University, Hanoi, Vietnam \\ Faculty of Applied Sciences, Delft University of Technology, \\ Julianalaan 136, 2826 BL, Deflt, The Netherland
}

\section{Eduado Mendes}

Faculty of Applied Sciences, Delft University of Technology, Julianalaan 136, 2826 BL, Deflt, The Netherland

\begin{abstract}
Polymer gels which were synthesized from acrylic acid and N-N' methylene bisacrylamide exhibited electrical sensitive behavior. Their swelling properties and electroresponsive behavior were studied. The results indicated that the water take-up ability of the hydrogel increased with the decreased N-N' methylene bisacrylamide content within the network. The hydrogel membranes swollen in a neutral $\mathrm{NaCl}$ solution, bent toward the cathode under non-contact $\mathrm{DC}$ electric fields, and their bending speed and equilibrium strain increased with the increased of applied voltage. In addition, the effect of crosslinker concentration of N-N' methylene bisacrylamide on bending behavior of the gels have been studied. The equilibrium strain decreased as the crosslinker concentration increased. By changing the direction of the applied potential cyclically, the hydrogel membranes exhibited good reversible bending behavior. The bending of the hydro gel membranes was initially explained by a bending theory of polyelectrolyte hydrogel based on the charge of osmotic pressure due to the ion concentration different between the inside and the outside of the network.
\end{abstract}

\section{INTRODUCTION}

Polymer gels consist of a crosslinked polymer network and a solvent. The network of long polymer molecules holds the liquid in place and so gives the gel what solidity it has.

A polymer gel is a soft and wet material capable of undergoing large deformation. A deformation gel, in turn, charges its chemical potential, behaving as energy transducer. Thus, a

\footnotetext{
*Corresponding author e-mail: namnph@vnu.edu.vn
} 
polymer gel shows a variety of stimuli-responsive actions, responding to external environmental changes.

It is well known that polymer gel in a solution swell or deswell when subjected to external stimuli such as change in $\mathrm{pH}$ and temperature, or light. Polyelectrolyte gels show the abilities of changing volume and shape reversibly in response to environment stimulus as temperature [1, 2], ionic strength [3], magnetic filed [4], and electric field [5 - 7], or light [8]. They have attracted much attention not only because of their unique properties but also because of their potential for significant technological and biomedical applications [9 - 11]. Among all intelligent hydrogels, electric field responsive hydrogel seems to be particularly interesting in connection with the fact that the electric field is the most conventional and convenient stimulus from the point of signal control. Electric field induced bending deformation or volume change of polyelectrolyte gels can transform chemical free energy directly into mechanical work to give isothermal energy convention, and can be used as actuators, electromechanical engines, artificial muscles [12 - 14], chemical valves [10], and controlled drug delivery devices [15, 16].

A typical function of a gel containing ionic groups is to bend reversibly under the influence of an electric field, making it useful in some actuators driven by an electric field. From the viewpoint of mechanical engineering, great hopes are set on these materials as new actuators, especially in the field of robotics and medical welfare instruments. In fact, the realization of lightweight, soft touch, and powerful actuators is an important subject. Besides, the volume change has been utilized for solute permeation control through membranes, in electromechanical engines (energy conversion), and in controlled drug delivery.

In our previous studies [17], we investigated the static structure factor of weakly charged polyelectrolyte gels under uniaxial strain. Polymer concentration fluctuations in poly(acrylic acid) gels subjected to uniaxial elongation were studied using small angle neutron scattering (SANS). In the present work, polymer gels based on acrylic acid and $\mathrm{N}-\mathrm{N}$ ' methylene bisacrylamide were prepared by radical co-polymerization, and their swelling properties and bending behavior under $\mathrm{DC}$ electric field in neutral $\mathrm{NaCl}$ solution were studied.

\section{EXPERIMENTAL}

\subsection{Materials}

Acrylic acid (AA) monomer was purchased from Sigma-Aldrich Co. and used after purification by distillation at $40^{\circ} \mathrm{C} / 26 \mathrm{~mm} \mathrm{Hg}$. N-N' methylene bisacrylamide (MBA), ammonium persulfate (APS) and sodium hydroxide $(\mathrm{NaOH})$ were purchased from Sigma-Aldrich Co. and used as received.

\subsection{Synthesis of hydrogel}

Hydrogels composed of AA and MBA were prepared by free radical solution polymerization in the presence of a crosslinking agent MBA. The crosslinked hydrogels were prepared using various monomer ratios in $\mathrm{H}_{2} \mathrm{O}$ as show in Table 1. The monomer concentration in the reaction bath was $\mathrm{Cm}=0,092 \mathrm{~g} / \mathrm{cc}$, and the neutralization degree, $\mathrm{f}=[\mathrm{NaOH}] /[$ monomer $]$, was fixed by adding the appropriate amount of $\mathrm{NaOH}$ to shift the acido-basic equilibrium governing the dissociation of the weak poly-acid. The value of $f$ was taken to be $f=0,1$. After bubbling of argon through the initial solution, the reaction was initiated with APS ( $2 \% \mathrm{~g} / \mathrm{g})$. The solution was put into a mold and kept in an oven at $70^{\circ} \mathrm{C}$ for 24 hours. The resulting hydrogels were immersed in distilled water for three days to remove all water-soluble and unreacted moieties, and the water was replaced every $12 \mathrm{~h}$. Finally, the swollen hydrogels were dried at $40^{\circ} \mathrm{C}$ in a vacuum oven to constant weight. 
Table 1: Feed composition for the preparation of the polymer gels.

\begin{tabular}{llllll}
\hline \multirow{2}{*}{ Samples } & \multicolumn{5}{c}{ Components } \\
\cline { 2 - 6 } & $\mathbf{A A}(\mathbf{m o l})$ & $\mathbf{M B A}(\mathbf{m o l})$ & $\mathbf{N a O H}(\mathbf{g})$ & $\left(\mathbf{N H}_{4}\right)_{2} \mathbf{S}_{\mathbf{2}} \mathbf{O}_{\mathbf{8}}(\mathbf{g})$ & $\mathbf{H}_{\mathbf{2}} \mathbf{O}(\mathbf{m l})$ \\
\hline A & 1 & 0.025 & 4.2 & 1.6 & 867.4 \\
B & 1 & 0.05 & 4.2 & 1.6 & 867.4 \\
C & 1 & 0.075 & 4.2 & 1.6 & 867.4 \\
D & 1 & 0.1 & 4.2 & 1.6 & 867.4 \\
E & 1 & 0.125 & 4.2 & 1.6 & 867.4 \\
\hline
\end{tabular}

\subsection{Swelling measurement}

The dry samples were immersed in distilled water at room temperature and the weight of the swollen samples were measured against time after excessive water on the surface of hydrogel membrane was removed using chemical qualitative analysis filter paper. The swelling ratio $(\mathrm{Sw})$ of each sample at time $t$ was calculated by the following equation:

$$
\mathrm{Sw}=(\mathrm{Wt}-\mathrm{Wd}) / \mathrm{Wd}
$$

where Wt and Wd were the weights of samples at time $t$ and in the dry state, respectively.

\subsection{Electroresponsive behavior measurement}

The hydrogel membrane was swollen in a $0,15 \mathrm{~mol} / \mathrm{l} \mathrm{NaCl}$ solution and reached its equilibrium state at room temperature and was cut into $12 \times 1,0 \times 0,5(\mathrm{~mm})$ strips. One end of the sample strip was fixed and placed vertically between two parallel electrodes in $\mathrm{NaCl}$ solution $0,15 \mathrm{~mol} / \mathrm{l}$ as showed in Figure 1.

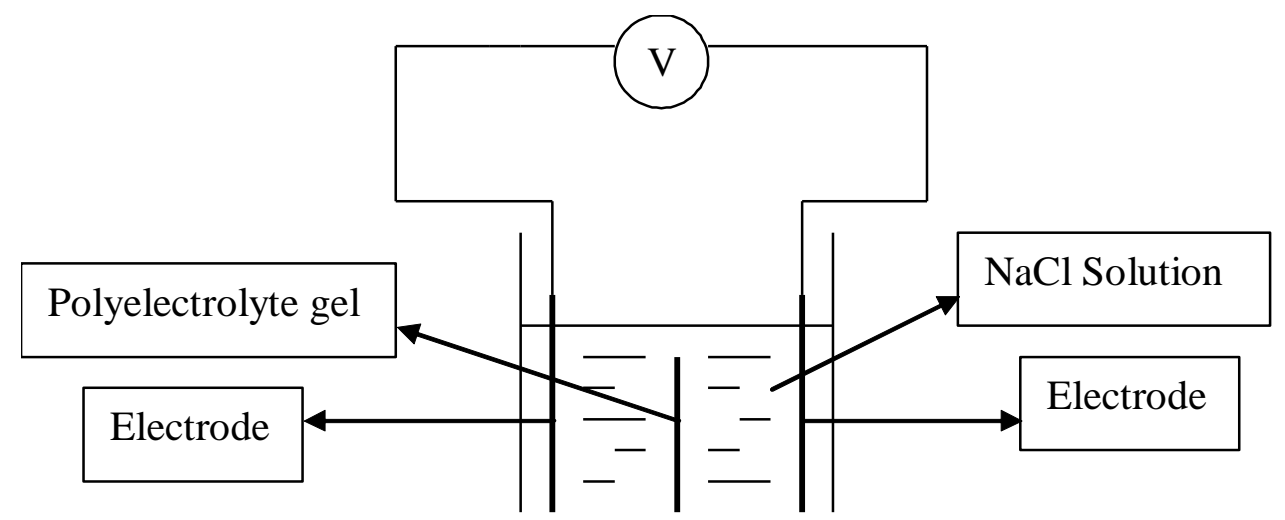

Fig. 1: Schematic illustration of measurement under dc electric field.

The distance between the two electrodes was $32 \mathrm{~mm}$. The bending deformation was recorded and measured by a video camera (Sony, japan). The deflection of bending is express in term of the distance between the ends of a hydrogel before and after bending, and the sign of the deflection is defined positive when a hydrogel bends to the cathode. The strain of bending $(\varepsilon)$ is calculated by Equation (1) [18]:

$$
\varepsilon=6 \mathrm{DY} / \mathrm{L}^{2}
$$


where $\mathrm{Y}$ is the deflection of bending, $\mathrm{D}$ is the thickness of the hydrogel and $\mathrm{L}$ is the original length of the hydrogel before application of electric field.

To test the reversible bending behavior of the hydrogel membranes, the polarity of the electric field was altered every $90 \mathrm{sec}$ by changing a two-way switch, and the bending deformation was recorded and measured by the video camera.

\section{RESULTS AND DISCUSSION}

\subsection{Swelling behavior of the polymer gels}

The swelling curves of the hydrogel as a function of time in distilled water are given in Figure 2.

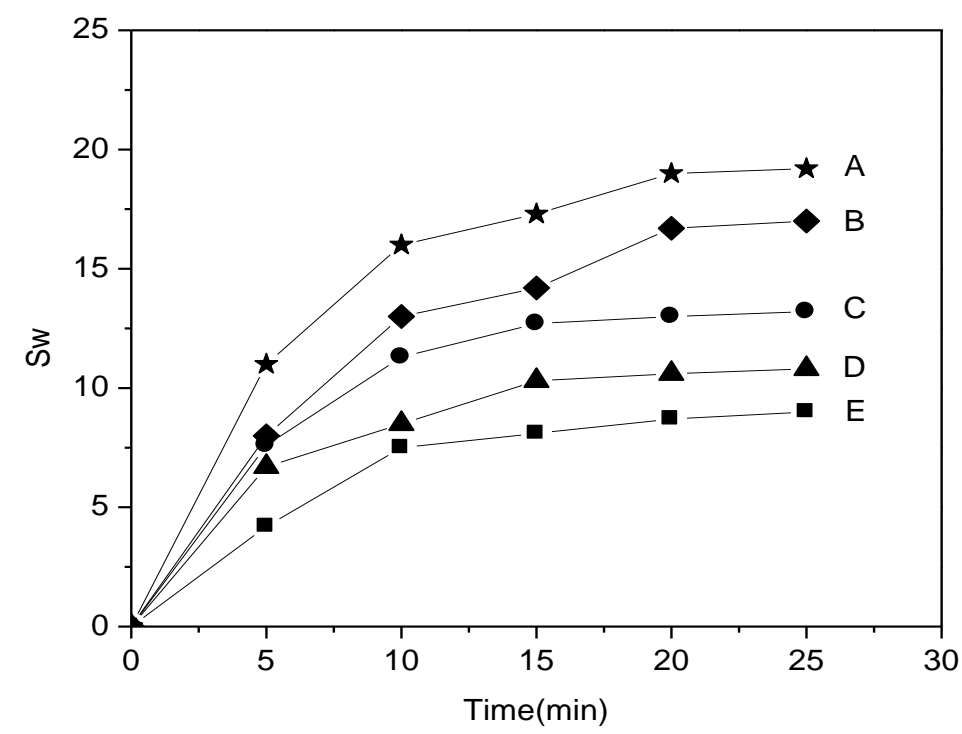

Fig. 2: Swelling kinetics of the hydro gel membranes measured as a function of time in distilled water.

The results indicated that the swelling ratio of the polymer gels decreased with the increasing of MBA monomer due to the increasing of the crosslinked agents in the polymeric network of the gel. The swelling ratio of the polyelectrolyte gel is determined by two major forces, that is, the electrostatic repulsion between the carboxylic acid polymer side chain and ions present in the solution, and the ionic osmotic pressure generated from mobile counterions to charged ions in the network (Donnan equilibrium) [19]. These two forces are influenced by the ionic strength of solution. An increase of ionic strength shields the charged groups in the network and reduces the Donnan effect, so the degree of swelling is decreased by the weakened electrostatic repulsion and the lowered ionic osmotic pressure. Ionic strength of the external solution increases with an amount of AA dissolved in one, i.e. unit mole ratio, $[\mathrm{AA}] /[\mathrm{MBA}]$. Therefore, swelling ratio of the gel decreases with an increase of [MBA].

\subsection{Electroresponsive behavior of the polymer gels}

The electroresponsive behavior of gels under DC electric field is shown in Figure 3. 


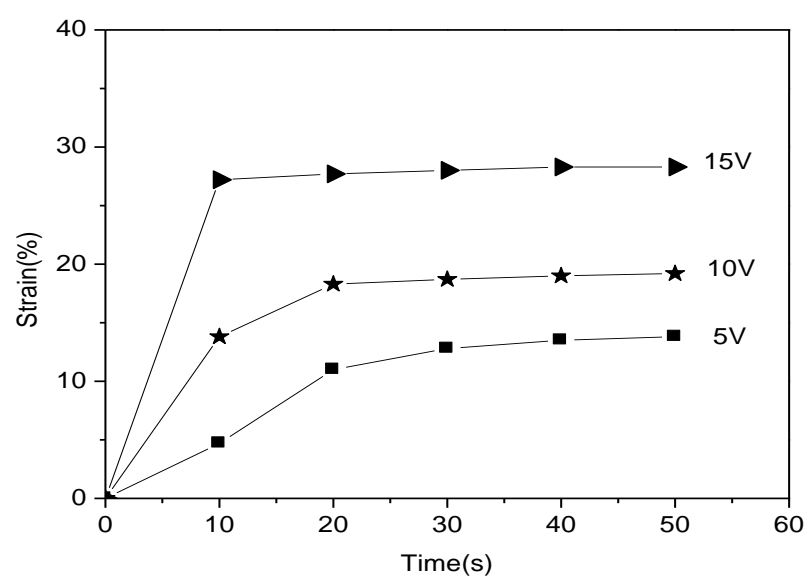

Fig. 3: Strain of the sample B as a function of time under different applied voltages in $\mathrm{NaCl}$ solution.

When the hydrogel sample in the $\mathrm{NaCl}$ electrolyte solution is subjected to an electric field, it bends toward the cathode. Figure 3 shows the relationship between the strain of sample B as a function of time and the voltage of the applied electric field in $\mathrm{NaCl}$ solution at room temperature. From Fig. 3 can see that, the degree of slope in the plot of the strain of bending versus time became steeper with increasing applied voltage and then leveled of at a steady state. The equilibrium strain increases with the applied voltage up to $15 \mathrm{~V}$. When it is more than $15 \mathrm{~V}$, the maximum strain is kept constant.

Shiga explained that the bending deformation of a polyelectrolytic hydrogel is induced by a change in the osmotic pressure based on a difference in mobile ion concentration between the inside and the outside of the gel [20]. When an electric field is applied on the negatively charged hydrogel in the aqueous solution, the counterion of the polyion, which is an ionic group in the polymer network, moves toward the negative electrode, while the polyion remain immobile. Also, the free ions in the surrounding solution move toward their counter-electrodes and come into the hydrogel. Thus, the osmotic pressure of the hydrogel polymer network near the positive electrode increases and becomes larger than that of the negative electrode side. Consequently, the osmotic pressure difference occurs within the hydrogel, and it is the driving force of bending toward the negative electrode. On the contrary, the positively charged hydrogel in the aqueous solution will bend toward the positive electrode under an DC electric field. Under the experimental conditions, the electrolytic solution is neutral $\mathrm{NaCl}$ solution about $\mathrm{pH} 7.0$, the polymer gels based on AA and MBA exist as polyanionic because of the dissociation of carboxyl, so the gels bend to the cathode under DC electric field.

In the bending of a polyelectrolytic hydrogel in aqueous solution with low electrolyte content, the osmotic pressure difference, $\Delta \pi$ is given approximately by a simple model expressed based on Donnan equilibrium:

$$
\begin{aligned}
& \Delta \pi=2 \mathrm{RTc}_{\mathrm{p}}\left(\mathrm{V}_{2} / \mathrm{V}_{1}\right) \text { ht }(1-\mathrm{ht}) \quad \text { (polyanion gel) } \\
& \Delta \pi=-2 \mathrm{RTc}_{\mathrm{p}}\left(\mathrm{V}_{2} / \mathrm{V}_{1}\right) \text { ht (1-ht) } \quad \text { (polycation gel) }
\end{aligned}
$$

where $\mathrm{R}$ is the gas constant, $\mathrm{T}$ is the absolute temperature, $\mathrm{c}_{\mathrm{p}}$ is the concentration of the polyions, $V_{2}$ and $V_{1}$ are the volumes of hydrogel and surrounding solution, $h$ is the transport rate of the counterion of the polyion from hydrogel to solution, or from solution to hydrogel, and $t$ is the time of applying an electric field, o $<$ ht $<1$. 
Equation (2) and (3) show that the polyion plays an important role in the bending and that nonionic gel doesn't show bending in DC electric fields.

From Fig. 3, note that the bending speed and equilibrium strain increase with the applied voltage, which could be explained by the fact that there was an enhancement in the transfer rate of the counterions of the immobile carboxylate groups on the polymer network from the hydrogel to the external solution and the free ions moved from the external solution into the hydrogel as the potential gradient in electric field is increased.

The reversible bending behavior of the hydrogel membranes depending on the electric stimulus was studied in $\mathrm{NaCl}$ solution. When an electric field was applied, the strip of the gel started to bend toward the cathode. When the electric stimulus was removed, the hydrogel was displaced to its original position. Also, if the polarity of the electric field was altered, the deformed hydrogel can be recovered to its original shape and then bent toward the opposite direction. Figure 4 shows a good reversible behavior of the sample B under a cyclically varying electric field from $10 \mathrm{~V}$ to $-10 \mathrm{~V}$ every $90 \mathrm{sec}$.

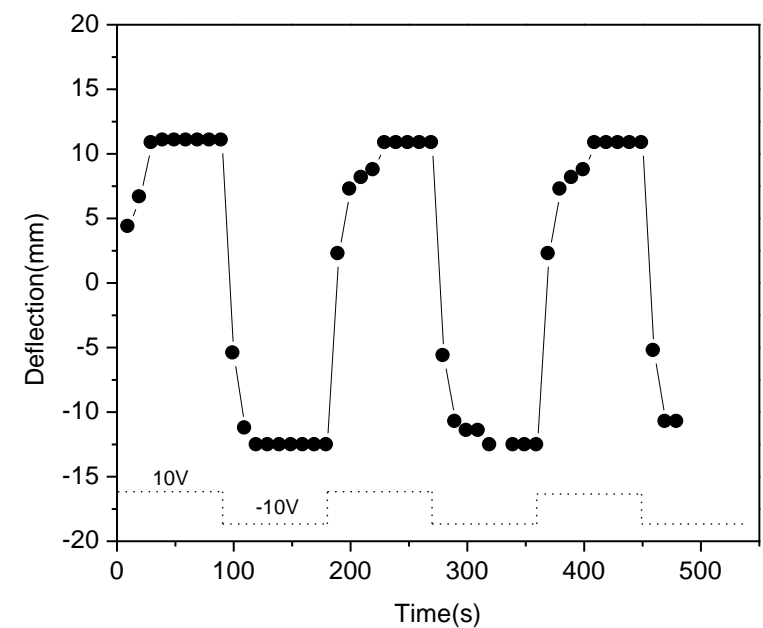

Fig. 4: Response of bending for the strip of sample B under a cyclically electric field from $10 \mathrm{~V}$ to $-10 \mathrm{~V}$.

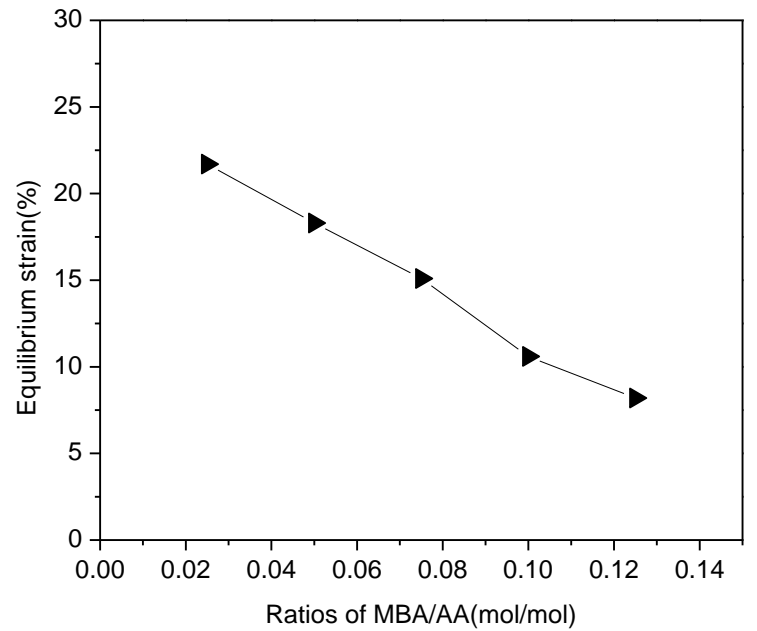

Fig. 5: Effect of crosslinker concentration (MBA) on the equilibrium strain of bending in $\mathrm{NaCl}$ solution under $10 \mathrm{~V}$ constant voltage. 
Figure 5 shows the effect of the crosslinker concentration (MBA) on the equilibrium strain of bending in $\mathrm{NaCl}$ solution at $10 \mathrm{~V}$.

From Fig. 5, the equilibrium strain decreased as the MBA concentration increased. Generally, the higher the crosslinker concentration, the higher the modulus of the hydrogel membrane, as the mobility of the chains were somewhat restricted because of the crosslinkage. With higher modulus, the hydrogel membrane deformation became more difficult. This was the reason the equilibrium strain decreased as the concentration increased.

In conclusion, the polymer gels based on acrylic acid (AA) and N-N' methylene bisacrylamide (MBA) were prepared by radical co-polymerization. The water take-up ability of the hydrogel membrane increased with the decreasing of crosslinked content within the net- work. Under an applied electric field, the polymer gels showed electromechanochemical phenomena. The bending behavior can be modulated via voltage of the applied electric field and the concentration of MBA within the hydrogel network. By changing the direction of the applied potential repeatedly, the gels exhibited good reversible bending behavior.

\section{REFERENCES}

1. Liang, L., Feng, X.D., Martin, P.F.C., and Peurrung, L.M. (2000), J. Appl. Polym. Sci., 75, pp. 1735-1739.

2. Chen, J. and Park, K. (1999), J. Macromol. Sci., Pure and Appl. Chem., A 36 (7\&8), pp. 917-930.

3. Zhang, R.S., Tang, M.G., Bowyer, A., Eisenthal, R., and Hubble (2005), J. Biomaterials, 26(22), pp. 4677-4683.

4. Zrinyi, M. and Szabo, D. (2001), Int. J. Mod. Phys. B, 15(6\&7), pp. 557-563.

5. Tanaka, T., Nishio, I., Sun, S.T., and Ueno-Nishio, S. (1982), Science, 218, pp. 467-469.

6. Grimshaw, P.E., Nussbaum, J.H., Grodzinsky, A.J., and Yarmush, M.L. (1990), J. Chem. Phys., 93(6), pp. 4462-4472.

7. Kim, S.J., Lee, K.J., Kim, S.L., Lee, Y.M., and Chung, T.D. (2003), J. Appl. Polym. Sci., 89, pp. 2301-2305.

8. Mamada, A., Tanaka, T., Kungwatchakun, D., and Irie, M. (1990), Macromolecules, 23, pp. 1517-1519.

9. Ito, Y. and Park, Y.S. (2000), Polym. Adv. Technol., 11, pp. 136-144.

10. Osada, Y. and Ross-Murphy, S.B. (1993), Sci. Am., 268(5), pp. 482-487.

11. Osada, Y., and Gong, J.P. (1998), Adv. Mater., 10(11), pp. 827-837.

12. Schreyer, H.B., Gebhart, N., Kim, K.J., and Shahinpoor, M. (2000), Biomacromolecules, 1, pp. 642-647.

13. James, V.L. (2002), Poptronics, 3(3), pp. 29-33.

14. Sun, S., and Mak, A.F.T. (2001), J. Polym. Sci. Part B, 39, pp. 236-246.

15. Kagatani, S., Shinoda, T., Konno, Y., Fukui, M., Ohmura, T., and Osada, Y. (1997), J. Pharm. Sci., 11, pp. 1273-1277.

16. Kim, S.Y. and Lee, Y.M. (1999), J. Appl. Polym. Sci., 74 (4), pp. 1752-1761.

17. Mendes, E., Schosseler, F., Isel, F., Boue, F., Bastide, J., and Candau, S.J. (1995), Europhys. Lett., 28, pp. 212-217. 
18. Shiga, T., Hirose, Y., Okada, A., and Kurauchi, T. (1992), J. Appl. Polym. Sci., 14(2), pp. 249-253.

19. Yoo, M.K., Sung, Y.K., Lee, Y.M., and Cho, C.S. (2000), J. Polym., 41, pp. 5713-5719.

20. Shiga, T., and Kurauchi, T. (2000), J. Appl. Polym. Sci., 39(11\&12), pp. 2305-2320. 